OPEN ACCESS

Edited by:

P. Bryant Chase,

Florida State University, USA

Reviewed by:

Marion Lewis Greaser,

University of Wisconsin-Madison, USA

Norio Fukuda,

Jikei University School of Medicine,

Japan

*Correspondence:

Bertrand C. W. Tanner btanner@vetmed.wsu.edu

Specialty section:

This article was submitted to Striated Muscle Physiology, a section of the journal

Frontiers in Physiology

Received: 10 May 2016 Accepted: 14 July 2016 Published: 29 July 2016

Citation:

Pulcastro HC, Awinda PO, Methawasin M, Granzier H, Dong W and Tanner BCW (2016) Increased

Titin Compliance Reduced

Length-Dependent Contraction and Slowed Cross-Bridge Kinetics in

Skinned Myocardial Strips from $R b m^{20} \Delta R R M$ Mice.

Front. Physiol. 7:322

doi: 10.3389/fphys.2016.00322

\section{Increased Titin Compliance Reduced Length-Dependent Contraction and Slowed Cross-Bridge Kinetics in Skinned Myocardial Strips from $\boldsymbol{R b m}^{20 \Delta R R M}$ Mice}

\author{
Hannah C. Pulcastro ${ }^{1}$, Peter O. Awinda ${ }^{1}$, Mei Methawasin ${ }^{2}$, Henk Granzier $^{2}$, \\ Wenji Dong $^{1,3}$ and Bertrand C. W. Tanner ${ }^{1 *}$
}

\begin{abstract}
${ }^{1}$ Department of Integrative Physiology and Neuroscience, Washington State University, Pullman, WA, USA, ${ }^{2}$ Department of Cellular and Molecular Medicine, University of Arizona, Tucson, AZ, USA, ${ }^{3}$ Voiland School of Chemical Engineering and Bioengineering, Washington State University, Pullman, WA, USA
\end{abstract}

Titin is a giant protein spanning from the Z-disk to the M-band of the cardiac sarcomere. In the I-band titin acts as a molecular spring, contributing to passive mechanical characteristics of the myocardium throughout a heartbeat. RNA Binding Motif Protein 20 (RBM20) is required for normal titin splicing, and its absence or altered function leads to greater expression of a very large, more compliant N2BA titin isoform in $R b m 20$ homozygous mice $\left(R b m 20^{\Delta R R M}\right)$ compared to wild-type mice (WT) that almost exclusively express the stiffer N2B titin isoform. Prior studies using $R b m 20^{\Delta R R M}$ animals have shown that increased titin compliance compromises muscle ultrastructure and attenuates the Frank-Starling relationship. Although previous computational simulations of muscle contraction suggested that increasing compliance of the sarcomere slows the rate of tension development and prolongs cross-bridge attachment, none of the reported effects of $R b m 20^{\Delta R R M}$ on myocardial function have been attributed to changes in cross-bridge cycling kinetics. To test the relationship between increased sarcomere compliance and cross-bridge kinetics, we used stochastic length-perturbation analysis in $\mathrm{Ca}^{2+}$-activated, skinned papillary muscle strips from Rbm20 $\triangle R R M$ and WT mice. We found increasing titin compliance depressed maximal tension, decreased $\mathrm{Ca}^{2+}$-sensitivity of the tension-pCa relationship, and slowed myosin detachment rate in myocardium from $R b m 20^{\triangle R R M}$ vs. WT mice. As sarcomere length increased from 1.9 to $2.2 \mu \mathrm{m}$, length-dependent activation of contraction was eliminated in the $R b m 20^{\triangle R R M}$ myocardium, even though myosin MgADP release rate decreased $\sim 20 \%$ to prolong strong cross-bridge binding at longer sarcomere length. These data suggest that increasing N2BA expression may alter cardiac performance in a length-dependent manner, showing greater deficits in tension production and slower cross-bridge kinetics at longer sarcomere length. This study also supports the idea that passive mechanical characteristics of the myocardium influence ensemble cross-bridge behavior and maintenance of tension generation throughout the sarcomere.

Keywords: cross-bridge kinetics, titin compliance, length-dependent activation, Frank-Starling relationship, cardiac muscle contraction 


\section{INTRODUCTION}

Titin is the largest protein that has been identified, spanning from the Z-disk to the M-band of the cardiac sarcomere (LeWinter et al., 2007). Acting as a molecular spring in the I-band, titin contributes to passive tension as sarcomeres are stretched and influences diastolic suction, or elastic recoil at short sarcomere lengths (Granzier and Irving, 1995; Helmes et al., 1996; Wu et al., 2000). Titin compliance is primarily dependent upon differential splicing, resulting in isoforms of different lengths (Labeit and Kolmerer, 1995; Freiburg and Gautel, 1996; Wu et al., 2000). RNA Binding Motif Protein 20 (RBM20) suppresses differential titin splicing such that wild-type mice (WT) predominantly express the stiffer N2B titin isoform and homozygous $R b m 20^{\triangle R R M}$ mice express a very large, more compliant N2BA titin isoform (Guo et al., 2013; Li et al., 2013; Methawasin et al., 2014).

Actin-myosin cross-bridge behavior is regulated by intracellular $\left[\mathrm{Ca}^{2+}\right]$ and sarcomere length, both of which are constantly changing throughout the heartbeat (for reviews see Tobacman, 1996; Cooke, 1997; Gordon et al., 2000; Kobirumaki-Shimozawa et al., 2014). Previous studies have shown that increased N2BA expression reduces passive tension (Fukuda et al., 2003; Makarenko et al., 2004; Nagueh et al., 2004; Hanft et al., 2014) which can compromise maximal $\mathrm{Ca}^{2+}$-activated tension production and reduce $\mathrm{Ca}^{2+}$-sensitivity of the tension-pCa relationship (Fukuda et al., 2001, 2003; Hanft et al., 2014; Methawasin et al., 2014). Increased myocardial compliance in $R b m 20^{\triangle R R M}$ mice and rats also demonstrated an attenuated Frank-Starling response (Methawasin et al., 2014; Ait-Mou et al., 2016). We have recently shown that cross-bridge cycling kinetics slowed at longer sarcomere length due to slowing of MgATP binding and MgADP release (Tanner et al., 2015). This led to the hypothesis that increased sarcomeric compliance in $R b m 20^{\triangle R R M}$ hearts could affect cross-bridge cycling kinetics differently at short vs. long sarcomere lengths, which may provide an explanation for compromised myocardial function in $R b m 20^{\Delta R R M}$ vs. WT myocardium.

To test this hypothesis we measured tension-pCa relationships, and cross-bridge kinetics at 1.9 and $2.2 \mu \mathrm{m}$ sarcomere length in skinned papillary muscle strips from WT and $R b m 20^{\triangle R R M}$ mice. We found increased titin compliance in the $R b m 20^{\triangle R R M}$ strips resulted in decreased maximal tension,

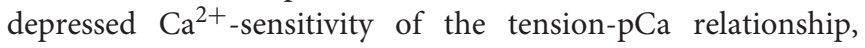
and slowed MgADP release compared to WT strips at each sarcomere length. As sarcomere length increased from 1.9 to $2.2 \mu \mathrm{m}$ sarcomere length, $R b m 20^{\triangle R R M}$ strips showed a minimal increase in maximal tension $\mathrm{Ca}^{2+}$-sensitivity of the tension-pCa relationship, while WT strips demonstrated a robust increase in tension and $\mathrm{Ca}^{2+}$-sensitivity of the tension pCa relationship. These findings suggest that titin compliance influences sarcomere-length dependent activation of contraction and cross-bridge nucleotide handling rates, influencing myocardial function more greatly at longer sarcomere length.

\section{MATERIALS AND METHODS}

\section{Animal Models}

All procedures were approved by the Institutional Animal Care and Use Committee at the University of Arizona and followed the U.S. National Institute of Health's "Using Animals in Intramural Research" guidelines for animal use. All mice were adult males, 25-32 weeks old. Wild-type (WT) mice were C57BL/6 strain. As previously characterized, exons 6 and 7 were deleted from the Rbm20 mouse gene to cause an in-frame deletion of the RNA Recognition Motif (RRM) that produced the $R b m 20^{\Delta R R M}$ genotype (Methawasin et al., 2014).

\section{Solutions for Skinned Myocardial Strips}

Muscle mechanics solution concentrations were formulated by solving equations describing ionic equilibria according to Godt and Lindley (1982), and all concentrations are listed in $\mathrm{mM}$ unless otherwise noted. Dissecting solution: $133.5 \mathrm{NaCl}, 5$ $\mathrm{KCl}, 1.2 \mathrm{NaH}_{2} \mathrm{PO}_{4}, 1.2 \mathrm{MgSO}_{4}, 30$ 2,3-butanedione monoxime (=BDM), 10 4-(2-Hydroxyethyl)piperazine-1-ethanesulfonic acid, N-(2-Hydroxyethyl)piperazine- $\mathrm{N}^{\prime}$-(2-ethanesulfonic acid; =HEPES; Methawasin et al., 2014). Skinning solution: 40 N,N-Bis(2-hydroxyethyl)-2-aminoethanesulfonic acid, $\mathrm{N}, \mathrm{N}$-Bis(2-hydroxyethyl)taurine (=BES), 10 Ethylene glycolbis(2-aminoethylether)- $\mathrm{N}, \mathrm{N}, \mathrm{N}^{\prime}, \mathrm{N}^{\prime}$-tetraacetic acid (=EGTA), 6.56 $\mathrm{MgCl}_{2}, 5.88$ ATP, 1 1,4-dithiothreitol (=DTT), $46.35 \mathrm{~K}$ propionate, 15 phosphocreatine, 0.4 Leupeptin, 0.1 transEpoxysuccinyl-L-leucylamido(4-guanidino)butane (=E-64), 0.5 Phenylmethanesulfonyl fluoride (=PMSF), $1 \%$ Triton $\mathrm{X}-100, \mathrm{pH} 7.0$ (Methawasin et al., 2014). Storage solution: 50 BES, $30.83 \mathrm{~K}$ propionate, 10 Na-azide, 20 EGTA, 6.29 ATP, 1 DTT, 20 BDM, $50 \mu \mathrm{M}$ Leupeptin, $275 \mu \mathrm{M}$ Pefabloc, and $1 \mu \mathrm{M}$ E-64 with 50\% glycerol wt/vol. Relaxing solution: pCa 8.0, 5 EGTA, $5 \mathrm{MgATP}, 1 \mathrm{Mg}^{2+}, 0.3 \mathrm{P}$, $20 \mathrm{BES}, 35$ phosphocreatine, $300 \mathrm{U} / \mathrm{mL}$ creatine kinase, 200 ionic strength adjusted with $\mathrm{Na}$ methanesulfonate, $\mathrm{pH}$ 7.0. Adding $0.3 \mathrm{mM}$ $\mathrm{P}_{\mathrm{i}}$ matches estimates for cardiac muscle ( $\mathrm{Wu}$ et al., 2008; Weiss et al., 2015), though others use higher [Pi] (Wang et al., 2014). Activating solution: Same as relaxing with pCa 4.8. Rigor solution: same as activating solution without MgATP.

\section{Skinned Myocardial Strips}

Left ventricular papillary muscles were dissected from the hearts of four WT mice and four $R b m 20^{\triangle R R M}$ mice $(\sim 180 \mu \mathrm{m}$ in diameter and $700 \mu \mathrm{m}$ long). Muscle strips were skinned in skinning solution overnight at $4^{\circ} \mathrm{C}$, and stored at $-20^{\circ} \mathrm{C}$ in storage solution for up to 1 week. Aluminum T-clips were attached to the end of each strip and strips were mounted between a piezoelectric motor (P841.40, Physik Instrumente, Auburn, MA) and a strain gauge (AE801, Kronex, Walnut Creek, CA), lowered into a $30 \mu \mathrm{L}$ droplet of relaxing solution maintained at $17^{\circ} \mathrm{C}$, and stretched to 1.9 or $2.2 \mu \mathrm{m}$ sarcomere length measured by digital Fourier Transform (IonOptix Corp, Milton, MA). 


\section{Dynamic Mechanical Analysis}

Stochastic length perturbations were applied for a period of $60 \mathrm{~s}$ as previously described (Tanner et al., 2011, 2015), using an amplitude distribution with a standard deviation of $0.05 \%$ muscle lengths over the frequency range $0.5-250 \mathrm{~Hz}$. Elastic and viscous moduli, $E(\omega)$ and $V(\omega)$, were measured as a function of angular frequency $(\omega)$ from the in-phase and out-of-phase portions of the tension response to the stochastic length perturbation. The complex modulus, $Y(\omega)$, was defined as $E(\omega)+i V(\omega)$, where $i=$ $\sqrt{ }-1$. Fitting Equation 1 to the entire frequency range of moduli values provided estimates of six model parameters $(A, k, B, 2 \pi b$, $C, 2 \pi c)$.

$$
Y(\omega)=A(i \omega)^{k}-B\left(\frac{i \omega}{2 \pi b+i \omega}\right)+C\left(\frac{i \omega}{2 \pi c+i \omega}\right) .
$$

The A-term in Equation (1) reflects the viscoelastic mechanical response of passive, structural elements in the muscle and holds no enzymatic dependence. The parameter $A$ represents the combined mechanical stress of the fiber, while the parameter $k$ describes the viscoelasticity of these passive elements, where $k=0$ represents a purely elastic response and $k=1$ is a purely viscous response (Mulieri et al., 2002; Palmer et al., 2013). The B- and C-terms in Equation (1) reflect enzymatic cross-bridge cycling behavior that produce frequency-dependent shifts in the viscoelastic mechanical response during $\mathrm{Ca}^{2+}$. activated contraction. These $\mathrm{B}$ - and C-processes characterize work-producing (cross-bridge attachment or recruitment) and work-absorbing (cross-bridge detachment) muscle responses, respectively (Kawai and Halvorson, 1991; Zhao and Kawai, 1993; Campbell et al., 2004; Palmer et al., 2007). The parameters $B$ and $C$ represent the mechanical stress from the cross-bridges (i.e., number of cross-bridges formed $\times$ their mean stiffness), and the rate parameters $2 \pi b$ and $2 \pi c$ reflect cross-bridge kinetics that are sensitive to biochemical perturbations affecting enzymatic activity, such as $[\mathrm{MgATP}],[\mathrm{MgADP}]$, or $\left[\mathrm{P}_{\mathrm{i}}\right]$ (Lymn and Taylor, 1971). Molecular processes contributing to crossbridge attachment or tension generation underlie the crossbridge attachment rate, $2 \pi b$. Similarly, processes contributing to cross-bridge detachment or tension decay underlie the crossbridge detachment rate, $2 \pi c$.

Stochastic system analysis provides a portrait of cross-bridge kinetics as a function of [MgATP]. Assuming that the myosin attachment events include time spent in the MgADP state and in the rigor state, the cross-bridge detachment rate can be described by:

$$
2 \pi c=\frac{k_{-A D P}[M g A T P]}{\frac{k_{-A D P}}{k_{+A T P}}+[M g A T P]} .
$$

As explained in detail by Tyska and Warshaw (2002) and implemented in our previous publications (Wang et al., 2013; Tanner et al., 2015), fitting the $2 \pi c-[\mathrm{MgATP}]$ relationship to Equation 2 allows a calculation of (i) $k_{-A D P}$, which represents cross-bridge MgADP release rate and the asymptotic, maximal myosin detachment rate in $\mathrm{s}^{-1}$ at saturating [MgATP]; and (ii) $k_{+A T P}$, which represents the second-order cross-bridge MgATP binding rate per myosin concentration in $\mathrm{M}^{-1} \mathrm{~s}^{-1}$.

\section{Statistical Analysis}

All values are shown as mean \pm SEM. Constrained non-linear least squares fitting of Equations $(1,2)$ to moduli was performed using sequential quadratic programming methods in Matlab ( $\mathrm{v}$ 7.9.0, The Mathworks, Natick MA). All statistical tests were performed using SPSS (IBM Statistics, Chicago, IL). A two-way ANOVA was used to assess effects of genotype and sarcomere length for parameter estimates from (i) the 3-parameter Hill fits to the tension-pCa relationships and (ii) the parameter estimates from fits to Equation (2) for the nucleotide handling rates. All other relationships were analyzed using linear mixed models with $\mathrm{pCa}$, frequency, or MgATP as a repeated measure, followed by a least significant difference post-hoc comparison of the means between genotype or sarcomere length. Statistical significance is reported at $p<0.05$.

\section{RESULTS}

There were no obvious differences in sarcomere organization or monitored sarcomere length in skinned papillary muscle strips from WT and $R b m 20^{\triangle R R M}$ mice (Figure 1A). As skinned myocardial strips were $\mathrm{Ca}^{2+}$-activated from $\mathrm{pCa} 8.0$ to $\mathrm{pCa} 4.8$, steady-state, isometric tension developed in a sigmoidal manner that was fit to a 3-parameter Hill equation (Figures 1B-F, Table 1). These tension-pCa relationships are shown two different ways, where: (i) where absolute tension values ( $=$ measured force values normalized to cross-sectional area of each myocardial strip; Figures 1C,D) illustrate the total tension produced by the strip (i.e., both the passive tension value at $\mathrm{pCa} 8.0$ plus the $\mathrm{Ca}^{2+}$-activated active tension values), and (ii) developed tension values illustrate the $\mathrm{Ca}^{2+}$-activated tension produced by the strip (i.e., absolute tension minus the passive, relaxed tension value at pCa 8.0; Figures 1E,F).

Under maximally activated conditions, myocardial strips with both WT and $R b m 20^{\triangle R R M}$ genotypes displayed greater absolute tension at 2.2 vs. $1.9 \mu \mathrm{m}$ sarcomere length (Figures 1C,D). Relaxed tension values ( $\mathrm{pCa} 8.0$ ) were also greater at the longer sarcomere length in both genotypes (Table 1). Developed tension was greater at 2.2 vs. $1.9 \mu \mathrm{m}$ sarcomere length from pCa 5.84.8 in myocardial strips from WT mice (Figure 1E). However, in myocardial strips from the $R b m 20^{\triangle R R M}$ mice, developed tension was only greater at $2.2 \mu \mathrm{m}$ sarcomere length at $\mathrm{pCa}$ 5.5 and 5.4 (Figure 1F). Thus, $\mathrm{Ca}^{2+}$-sensitivity of the tensionpCa relationship increased with sarcomere length in the WT strips (by $\sim 0.08$ pCa units), but this sarcomere length-dependent increase in $\mathrm{Ca}^{2+}$-sensitivity of tension was lost in $R b m 20^{\Delta R R M}$ strips (Table 1). At $2.2 \mu \mathrm{m}$ sarcomere length, WT strips also displayed greater $\mathrm{Ca}^{2+}$-sensitivity of tension than $\mathrm{Rbm} 20^{\triangle R R M}$ strips (by $\sim 0.07 \mathrm{pCa}$ units). In WT strips, the Hill coefficient $\left(\mathrm{n}_{\mathrm{H}}\right)$ for the tension-pCa relationship was smaller at 2.2 vs. 1.9 $\mu \mathrm{m}$ sarcomere length, indicating reduced cooperativity at longer sarcomere length (Table 1). At $2.2 \mu \mathrm{m}$ sarcomere length, $\mathrm{n}_{\mathrm{H}}$ was smaller for WT strips than Rbm20 $0^{\triangle R R M}$ strips; there were no differences in $\mathrm{n}_{\mathrm{H}}$ between genotypes at $1.9 \mu \mathrm{m}$ sarcomere length.

In both genotypes under relaxed conditions ( $\mathrm{pCa} 8.0$ ), elastic moduli values were greater at 2.2 vs. $1.9 \mu \mathrm{m}$ sarcomere length 
A
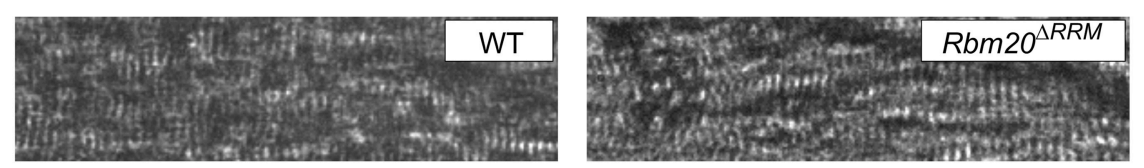

B
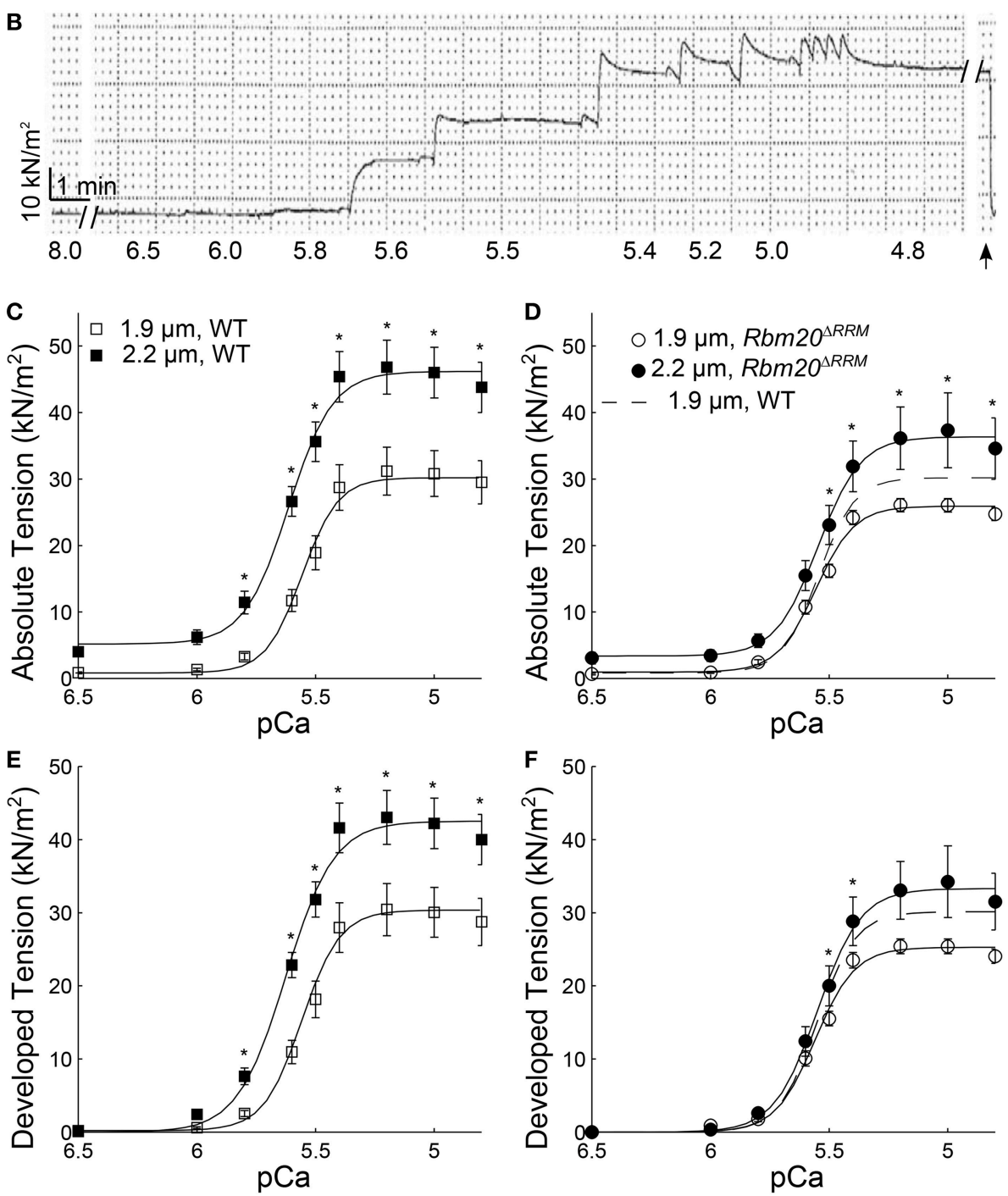

FIGURE 1 | Tension-pCa relationships at long vs. short sarcomere length. (A) Example light microscopy images at 40X, showing skinned myocardial strips from WT (left), and Rbm20 $\triangle R R M$ (right) mice. (B) An example absolute tension trace plotted against time from a myocardial strip that was Ca ${ }^{2+}$-activated from pCa 8.0 to 4.8 (pCa values listed below each solution exchange), where the strip was slacked (arrow) near the end of the experiment to ensure no baseline-tension changes throughout the time course of an experiment. Absolute tension-pCa relationships for (C) WT, and (D) $R b m 20^{\Delta R R M}$ mice and developed tension-pCa relationships for (E) WT and (F) $R b m 20^{\triangle R R M}$ mice at 1.9 and $2.2 \mu \mathrm{m}$ sarcomere length. Solid lines represent 3-parameter Hill fits to the tension-pCa data, with the dashed lines representing the $1.9 \mu \mathrm{m}$ sarcomere length fit for $R b m 20^{\Delta R R M}$ replotted in panel (D,F). ${ }^{\star} p<0.05$ between sarcomere length within a genotype.

for all frequencies $>1.5 \mathrm{~Hz}$ (Figures 2A,C). Viscous moduli values were also greater at longer sarcomere length at frequencies $>51 \mathrm{~Hz}$ in WT strips and frequencies $>54 \mathrm{~Hz}$ in $R b m 20^{\triangle R R M}$ strips (Figures 2B,C, respectively). Under activated conditions (pCa 4.8, $5 \mathrm{mM} \mathrm{MgATP),} \mathrm{elastic} \mathrm{moduli} \mathrm{values} \mathrm{were} \mathrm{greater}$ at longer sarcomere length for frequencies above $145 \mathrm{~Hz}$ in WT (Figure 3A), and frequencies $>22 \mathrm{~Hz}$ in $R b m 20^{\triangle R R M}$ strips (Figure 3C). In addition to these moduli differences, there was a consistent shift toward lower frequencies for the overall elastic moduli-frequency relationship at longer sarcomere length; this shift toward lower frequencies was larger for $R b m 20^{\Delta R R M}$ strips vs. WT strips. Under activated conditions, viscous moduli were not different at any particular sarcomere length in the WT strips (Figure 3B), and viscous moduli were greater at 2.2 vs. $1.9 \mu \mathrm{m}$ sarcomere length at frequencies between 9.5 and 54 $\mathrm{Hz}$ in the $R b m 20^{\triangle R R M}$ strips (Figure 3D). There was also a 
TABLE 1 | Characteristics of tension-pCa relationships in mouse myocardium at 1.9 and $2.2 \mu \mathrm{m}$ sarcomere lengths, with and without $R b m 20^{\Delta R R M}$ mutation (mean \pm SEM).

\begin{tabular}{|c|c|c|c|c|}
\hline & WT $1.9 \mu \mathrm{m}$ & WT $2.2 \mu \mathrm{m}$ & $\begin{array}{c}R b m 20^{\Delta R R M} \\
1.9 \mu m\end{array}$ & $\begin{array}{c}R b m 20^{\Delta R R M} \\
2.2 \mu \mathrm{m}\end{array}$ \\
\hline $\mathrm{T}_{\min }($ & $75 \pm 0.11$ & $.78 \pm 0.69^{*}$ & $0.66 \pm 0.11$ & $3.07 \pm 0.89^{*}$ \\
\hline $\mathrm{T}_{\max }\left(\mathrm{kN} / \mathrm{m}^{2}\right)$ & $29.50 \pm 3.26$ & $43.78 \pm 3.78^{\star}$ & $24.72 \pm 0.97$ & $34.58 \pm 4.62^{* \dagger}$ \\
\hline $\mathrm{T}_{\mathrm{dev}}\left(\mathrm{kN} / \mathrm{m}^{2}\right)$ & $28.75 \pm 3.23$ & $39.99 \pm 3.45^{\star}$ & $24.06 \pm 0.99$ & $31.51 \pm 3.88$ \\
\hline $\mathrm{pCa}_{50}$ & $5.55 \pm 0.01$ & $5.63 \pm 0.02^{*}$ & $5.56 \pm 0.01$ & $5.56 \pm 0.03^{\dagger}$ \\
\hline$n_{H}$ & $5.48 \pm 0.30$ & $4.48 \pm 0.20^{\star}$ & $5.43 \pm 0.27$ & $5.85 \pm 0.28^{\dagger}$ \\
\hline $\operatorname{Max}_{\mathrm{fit}}\left(\mathrm{kN} / \mathrm{m}^{2}\right)$ & $30.16 \pm 3.43$ & $42.46 \pm 3.59^{\star}$ & $25.27 \pm 1.02$ & $33.42 \pm 4.34^{\dagger}$ \\
\hline n fibers & 9 & 8 & 7 & 9 \\
\hline
\end{tabular}

$T_{\text {min }}$, absolute tension value at $p C a$ 8.0.

$T_{\text {max }}$, absolute tension value at $\mathrm{pCa} 4.8$.

$T_{\text {dev }}, \mathrm{Ca}^{2+}$-activated, developed tension $\left(T_{\max }-T_{\min }\right)$.

Max ${ }_{\text {fit }}, p C a_{50}$, and $n_{H}$ represent fit parameters to a 3-parameter Hill equation for the

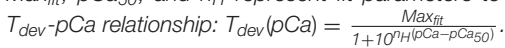

${ }^{\dagger} p<0.05$ effect of mutation at same sarcomere length.

${ }^{*} p<0.05$ effect of sarcomere length within a mutation/genotype.

consistent shift toward lower frequencies for the overall viscous moduli-frequency relationship at longer sarcomere length; this shift toward lower frequencies was larger for $R b m 20^{\triangle R R M}$ strips vs. WT strips. Altogether these data indicate greater myocardial viscoelasticity at longer sarcomere length under relaxed and activated conditions, although the influence of titin compliance was minimal as there were no significant effects of genotype in the moduli-frequency relationships (Figures 2, 3). The length-dependent shifts toward lower frequencies in the moduli-frequency relationships at $\mathrm{pCa} 4.8$ indicate slower cross-bridge cycling as sarcomere length increased for both genotypes, although this slowing was greater for $R b m 20^{\Delta R R M}$.

Moduli values were fit to Equation (1) to extract model parameters related to viscoelasticity, cross-bridge binding, and cross-bridge kinetics as the skinned strips were titrated toward rigor (5.0-0.05 mM MgATP, pCa 4.8). These model parameters are plotted against [MgATP] in Figure 4, with $p$-values listed in the left panel for each parameter that demonstrated significant main effects or interactions from the mixed-model analysis. As [MgATP] was titrated toward rigor, $A$ values increased and $k$ values decreased for both genotypes, suggesting increased viscoelastic myocardial stiffness that became more elastic (vs. viscous) due to greater cross-bridge binding as MgATP decreased (Figures 4A-D). In both genotypes, $A$ values were greater and $k$ values were smaller at 2.2 vs. $1.9 \mu \mathrm{m}$ sarcomere length, which represents greater myocardial viscoelasticity due to a combination of: (i) passive elements of the sarcomere being stretched or extended more at 2.2 vs. $1.9 \mu \mathrm{m}$ sarcomere length and (ii) greater binding of slower-cycling cross-bridges at $2.2 \mathrm{vs.}$ $1.9 \mu \mathrm{m}$ sarcomere length. For both genotypes, the values for $B$ and $C$ increased as [MgATP] was titrated toward rigor and the magnitudes for $C$ increased at 2.2 vs. $1.9 \mu \mathrm{m}$ sarcomere length (Figures $4 \mathrm{E}, \mathrm{F}$ ), also suggesting greater cross-bridge binding at longer sarcomere length.

As $[\mathrm{MgATP}]$ decreased, cross-bridge attachment rate $(2 \pi \mathrm{b}$, Figures 4I,J) slowed in both genotypes. The significant MgATP $\times$ genotype interaction suggests cross-bridge attachment rate was more sensitive to [MgATP] in WT than in Rbm20 ${ }^{\triangle R R M}$ strips, although cross-bridge attachment rates were not different at 2.2 vs. $1.9 \mu \mathrm{m}$ sarcomere length. Similarly, as [MgATP] decreased toward rigor, cross-bridge detachment rate $(2 \pi \mathrm{c}$, Figures $4 \mathrm{~K}, \mathrm{~L})$ slowed in both genotypes. Cross-bridge detachment rates were also slower at $2.2 \mathrm{vs.} 1.9 \mu \mathrm{m}$ sarcomere length for both genotypes. The significant genotype effect on cross-bridge detachment rate suggests that $R b m 20^{\triangle R R M}$ strips displayed a slower cross-bridge detachment rate than WT strips across the entire [MgATP] range, although this statistic was primarily driven by the slowest detachment rates occurring for $R b m 20^{\triangle R R M}$ strips at $2.2 \mu \mathrm{m}$ sarcomere length. Again the significant MgATP $\times$ genotype interaction suggests that cross-bridge detachment rate was more sensitive to [MgATP] in WT than in Rbm20 ${ }^{\triangle R R M}$ strips.

Fitting the $2 \pi \mathrm{c}$-MgATP relationship to Equation (2) (solid lines in Figures $4 \mathbf{K}, \mathbf{L}$ ) provides an estimate of the cross-bridge rates of MgADP release $\left(k_{-A D P}\right)$ and MgATP binding $\left(k_{+A T P}\right)$. The MgADP release rate slowed with increased titin compliance for the $R b m 20^{\triangle R R M}$ fibers, and there was a length-dependent slowing of $k_{-A D P}$ at longer sarcomere length for both genotypes (Table 2). For WT fibers, increasing sarcomere length from 1.9 to $2.2 \mu \mathrm{m}$ slowed MgADP release by $12 \%$ ( $p=0.015$ using a $t$-test). For $R b m 20^{\triangle R R M}$ fibers $k_{-A D P}$ slowed $22 \%$ as sarcomere length increased from 1.9 to $2.2 \mu \mathrm{m}$, showing about twice as much length-dependent slowing of $k_{-A D P}$ for $R b m 20^{\triangle R R M}$ than WT. However, increased titin compliance in the Rbm $20^{\triangle R R M}$ strips led to slower rates of MgADP release at both sarcomere lengths (13 and 23\% slower at short and long sarcomere length, respectively), compared to WT $k_{-A D P}$ values. The cross-bridge rate of MgATP binding did not differ with genotype or with sarcomere length (Table 2). These finding suggest that increased compliance of the myofilament lattice slows cross-bridge cycling kinetics, primarily due to slower MgADP dissociation from cross-bridges.

\section{DISCUSSION}

Computational simulations of muscle contraction have demonstrated that mechanical characteristics of the sarcomere (i.e., filament, cross-bridge, and titin compliance; compliance $=$ stiffness $^{-1}$ ) influence the dynamics of cross-bridge binding and tension generation in a muscle fiber (Daniel et al., 1998; Martyn et al., 2002; Chase et al., 2004; Campbell, 2006, 2009, 2016; Sheikh et al., 2012; Tanner et al., 2012a, 2014). These mathematical models predict that increasing sarcomeric compliance diminishes steady-state tension, slows the apparent rate of tension development, slows cross-bridge cycling rates, and can impact the rate of tension relaxation as well. As RMB20 $\triangle R R M$ mice express more of the compliant N2BA titin isoform than the WT (Guo et al., 2013; Methawasin et al., 2014), these transgenic animals represent a useful model system to test some of these model predictions and directly assess the role of titin compliance in length-dependent tension production and ensemble crossbridge behavior in skinned myocardial strips. In this study we observed that increased titin compliance in $R b m 20^{\Delta R R M}$ fibers diminished steady-state tension, reduced $\mathrm{Ca}^{2+}$-sensitivity of the 


\section{Relaxed (pCa 8.0)}
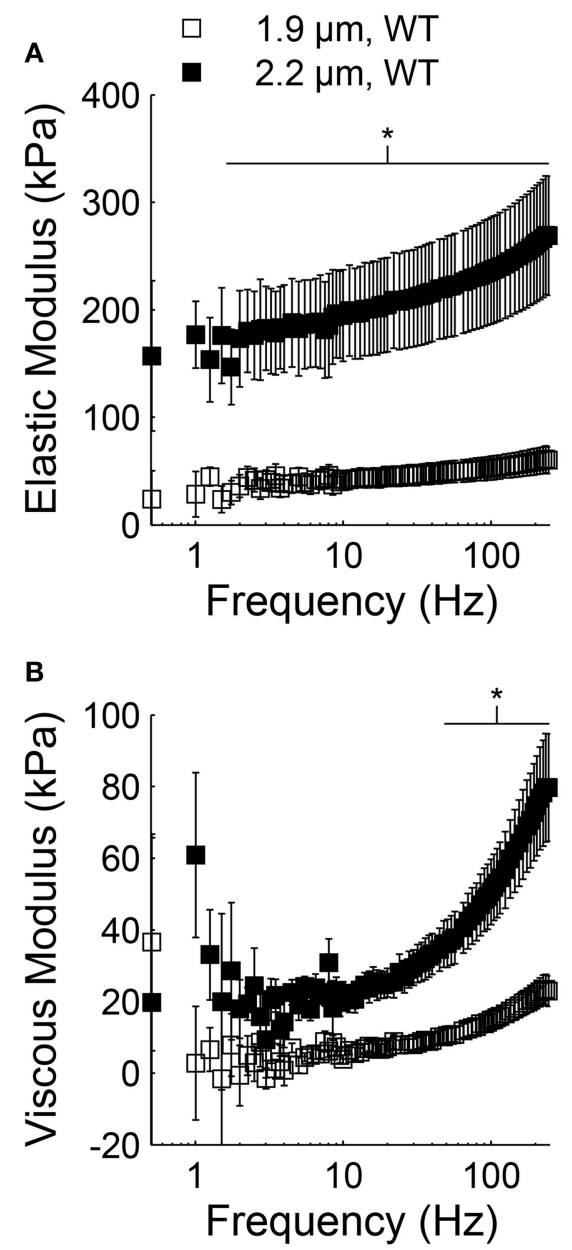

\section{Relaxed (pCa 8.0)}

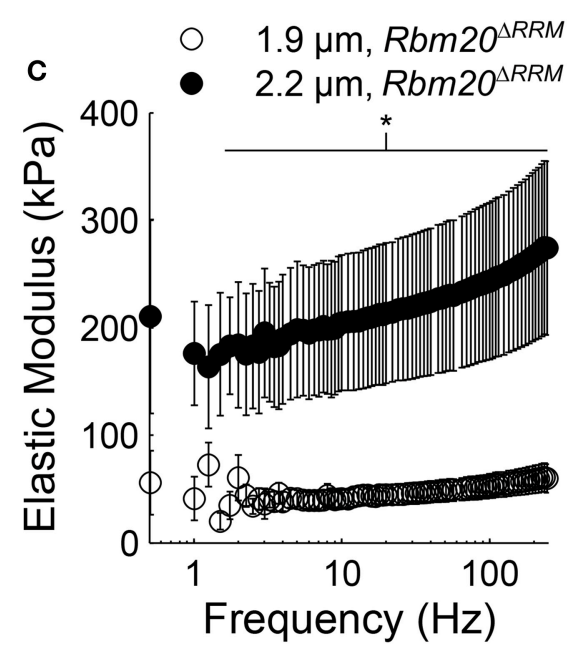

D

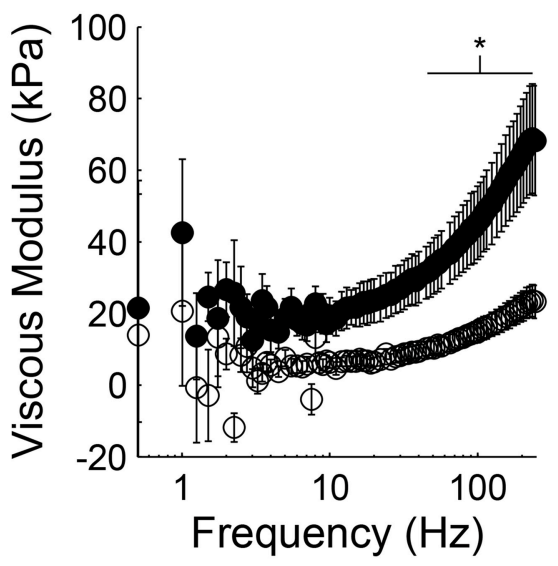

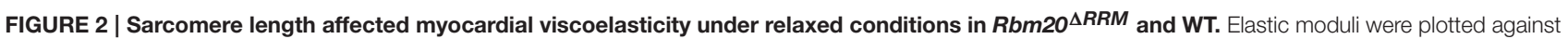
frequency for (A) WT and (C) $R b m 20^{\triangle R R M}$ genotypes for skinned papillary muscle strips at 1.9 and $2.2 \mu \mathrm{m}$ sarcomere lengths under relaxed conditions (pCa 8 and 5 mM MgATP). The associated viscous moduli were plotted against frequency for (B) WT and (D) Rbm20 $\Delta R R M$ at 1.9 and $2.2 \mu \mathrm{m}$ sarcomere lengths. ${ }^{*} p<0.05$ between sarcomere lengths within a genotype.

tension-pCa relationship, and slowed cross-bridge detachment rate due to slowed MgADP dissociation from strongly-bound cross-bridges. The effects of titin compliance were sarcomere length-dependent, showing almost no length-dependent tension response in $R b m 20^{\triangle R R M}$ strips, in contrast to the robust lengthdependent increase in maximal tension and $\mathrm{Ca}^{2+}$-sensitivity of the tension-pCa relationships between 1.9 and $2.2 \mu \mathrm{m}$ sarcomere length in WT strips. This length-dependent activation response was eliminated in $R b m 20^{\triangle R R M}$ strips despite a slowed cross-bridge detachment rate as sarcomere length increased, which would be expected to enhance thin-filament activation at $2.2 \mu \mathrm{m}$ sarcomere length due to strong cross-bridge binding (Bremel and Weber, 1972; Wang and Fuchs, 1994; Metzger, 1995; Fitzsimons and Moss, 1998; Smith et al., 2009; Terui et al., 2010; Li et al., 2014). Empirical findings in this study support previous computational simulations predicting the important role that sarcomeric compliance plays in muscle contraction and further suggests that titin mechanics affect length dependent activation of contraction, perhaps by altering how tension propagates throughout the sarcomere to influence thin-filament activation.

Our observations that increased titin compliance in the Rbm $20^{\triangle R R M}$ strips reduced maximal tension values and decreased $\mathrm{Ca}^{2+}$ sensitivity of tension agree with previous findings that suggest greater N2BA titin isoform expression depresses maximum tension production (Makarenko et al., 2004; Lewinter et al., 2010; Patel et al., 2012; Hanft et al., 2014; Methawasin et al., 2014). Our measurements also show that effects of titin compliance on $\mathrm{Ca}^{2+}$-activated tension are sarcomere length-dependent, supporting previous studies showing that increased titin compliance depresses tension more significantly at longer sarcomere length (Fukuda et al., 2003; Methawasin et al., 2014). This is most evident by the similar tension-pCa relationships at $1.9 \mu \mathrm{m}$ sarcomere length among both genotypes (Figure 1; Table 1), with a robust 


\section{Active (pCa 4.8)}

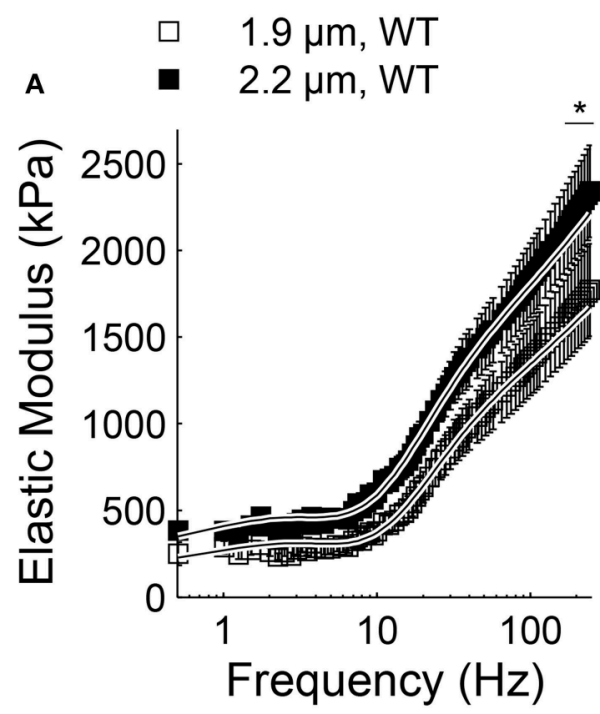

B

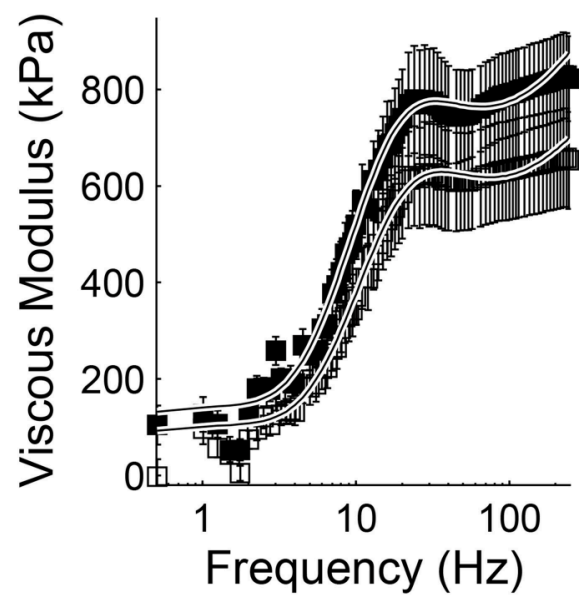

Active (pCa 4.8)

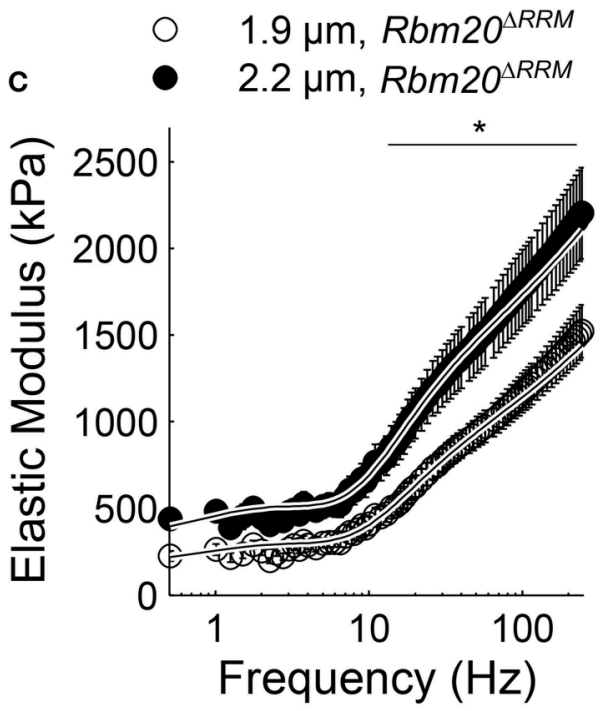

D

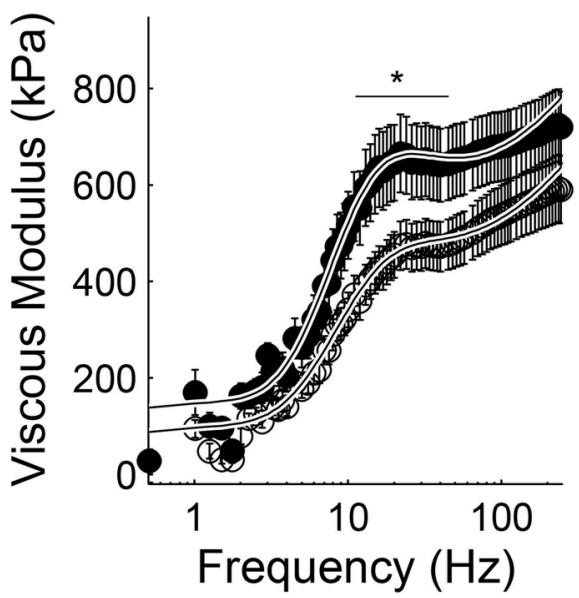

FIGURE 3 | Sarcomere length affected myocardial elasticity in Rbm20 $\mathbf{A R R M}$ and WT. Elastic moduli were plotted against frequency for (A) WT and (C) Rbm20 ${ }^{\Delta R R M}$ genotypes for skinned papillary muscle strips at 1.9 and $2.2 \mu \mathrm{m}$ sarcomere lengths under activated conditions (pCa 4.8 and 5 mM MgATP). The associated viscous moduli were plotted against frequency for (B) WT and (D) Rbm20 $\Delta R R M$ at 1.9 and $2.2 \mu \mathrm{m}$ sarcomere lengths. ${ }^{*} p<0.05$ between sarcomere lengths within a genotype.

length-dependent increase in $\mathrm{Ca}^{2+}$-activated tension production as sarcomere length increased to $2.2 \mathrm{um}$ for WT strips that did not occur for $R b m 20^{\triangle R R M}$ strips. These data suggest that length-dependent activation of contraction and the slope of the ascending limb of the sarcomere-length vs. $\mathrm{Ca}^{2+}$-activated tension relationship may depend upon mechanical characteristics of titin. This implies that dynamic processes related to crossbridge cycling kinetics, thin-filament activation, and tension development within the sarcomere may be influenced by the mechanical characteristics of titin.

Cross-bridge detachment rates slowed as sarcomere length increased from 1.9 to $2.2 \mu \mathrm{m}$ among both genotypes, but the slowing was more pronounced for the $R b m 20^{\triangle R R M}$ strips. For
WT strips, slower myosin detachment at $2.2 \mu \mathrm{m}$ sarcomere length effectively enhances cross-bridge contributions to thinfilament activation to augment tension production and $\mathrm{Ca}^{2+}$. sensitivity of the tension-pCa relationship. Previous studies have linked greater $\mathrm{Ca}^{2+}$-affinity of troponin $\mathrm{C}$ and greater opening of the $\mathrm{N}$-terminus of troponin $\mathrm{C}$ with increases in strong cross-bridge binding (Hofmann and Fuchs, 1987; Wang and Fuchs, 1994; Terui et al., 2008, 2010; Smith et al., 2009; Li et al., 2014), and our current findings in WT strips and rat papillary muscle strips (Tanner et al., 2015; Pulcastro et al., 2016) imply this cooperative activation pathway becomes stronger at longer sarcomere lengths. The MgADP release rate $\left(k_{-A D P}\right)$ was $13 \%$ slower at $1.9 \mu \mathrm{m}$ and $23 \%$ slower at 2.2 


\section{Active (pCa 4.8)}

$\begin{array}{ll}\square & 1.9 \mu \mathrm{m}, \mathrm{WT} \\ \square \quad 2.2 \mu \mathrm{m}, \mathrm{WT}\end{array}$
Active (pCa 4.8)

O $\quad 1.9 \mu \mathrm{m}, R b m 20^{\triangle R R M}$

- $\quad 2.2 \mu \mathrm{m}, R b m 20^{\triangle R R M}$
A

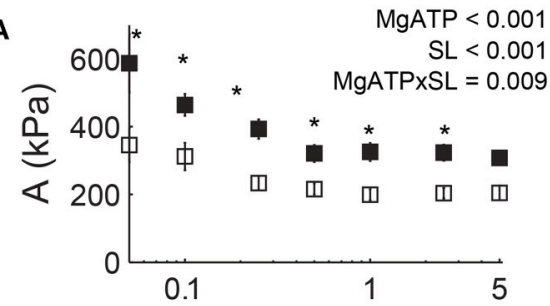

C

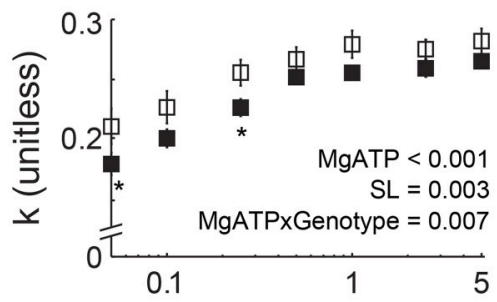

E

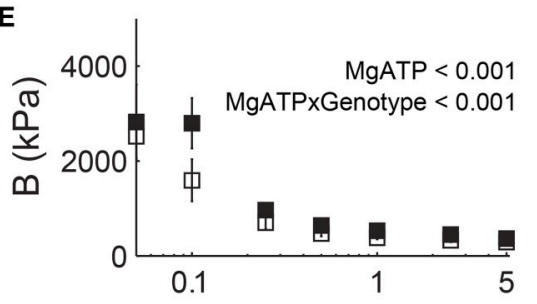

G
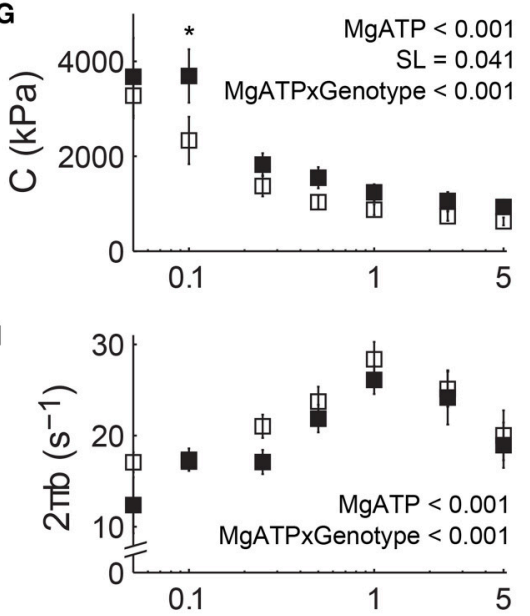

K

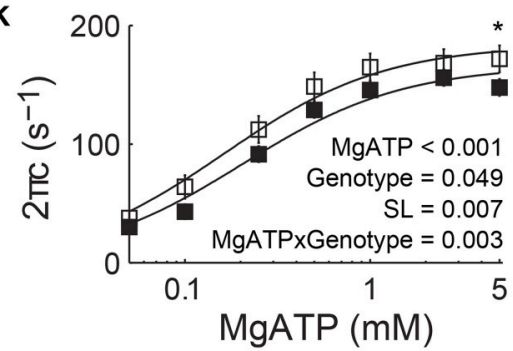

B

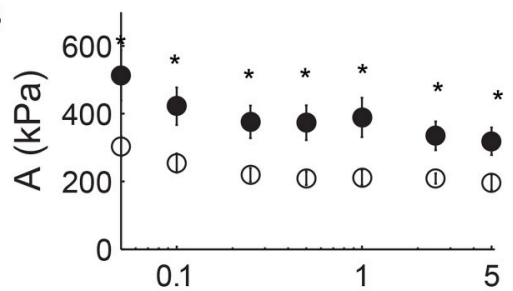

D

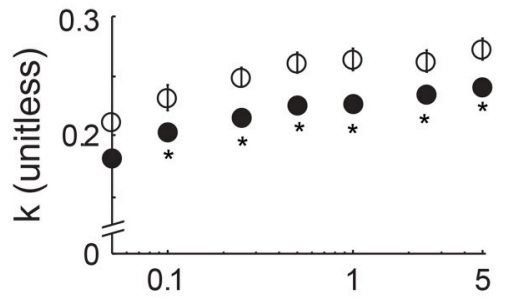

F
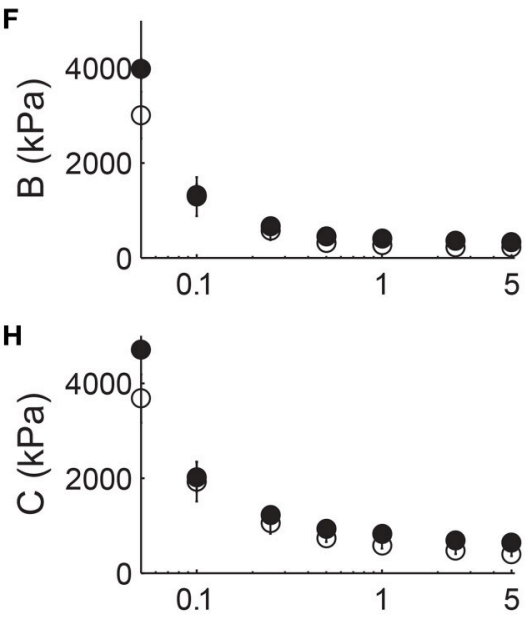

J

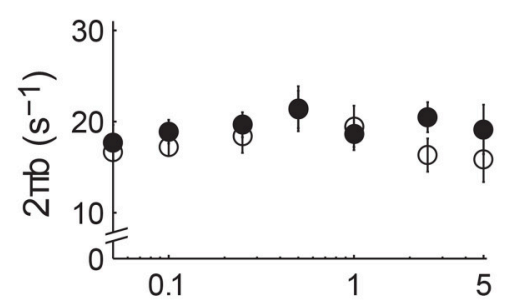

L

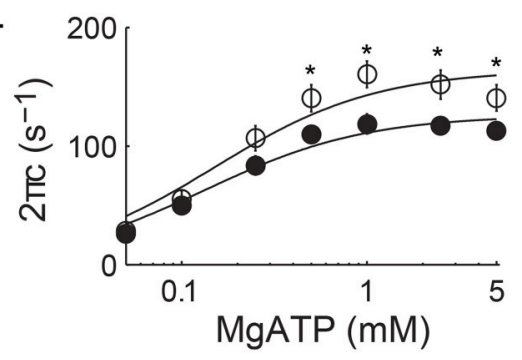

FIGURE 4 | Increased titin compliance affected myocardial viscoelasticity and cross-bridge kinetics as [MgATP] varied at pCa 4.8. Parameter fits to Equation (1) are plotted against [MgATP] at 1.9 and $2.2 \mu \mathrm{m}$ sarcomere lengths for skinned papillary muscle strips from WT (left set of panels) and Rbm20 $\Delta R R M$ (right set of panels) mice. Myocardial viscoelastic stiffness increased and became increasingly elastic as [MgATP] decreased, as reflected by the MgATP-dependent increase in $A(\mathbf{A}, \mathbf{B})$ and decrease in $k(\mathbf{C}, \mathbf{D})$. Magnitude parameters for the B-process $(\mathbf{E}, \mathbf{F})$ and the C-process $(\mathbf{G}, \mathbf{H})$ also increased as [MgATP] decreased, which 


\section{FIGURE 4 | Continued}

indicates an expected increase in cross-bridge binding as [MgATP] was titrated toward rigor. The rate of cross-bridge attachment, $2 \pi b$ (I,J), and the rate of cross-bridge detachment, $2 \pi \mathrm{c}(\mathbf{K}, \mathbf{L})$, decreased as [MgATP] decreased, which indicates the expected slowing of cross-bridge cycling kinetics as [MgATP] was titrated toward rigor. Dashed lines representing the $R b m 20^{\Delta R R M}, 1.9 \mu \mathrm{m}$ sarcomere length data were replotted in the left set of panels. $P$-values listed within the left panel show significant $(<0.05)$ main effects of [MgATP], genotype, sarcomere length $(\mathrm{SL})$, and any interactions between these effects among all four sets of data, resulting from mixed models analysis of each parameter-[MgATP] relationship. ${ }^{*} p<0.05$ between sarcomere lengths within a genotype.

TABLE 2 | Estimates of myosin cross-bridge kinetics from fits of the cross-bridge detachment rate (2 $\pi \mathrm{c})$ vs. MgATP relationships to Equation (2) for 1.9 and $2.2 \mu \mathrm{m}$ sarcomere lengths (mean \pm SEM).

\begin{tabular}{|c|c|c|c|c|}
\hline & WT $1.9 \mu \mathrm{m}$ & WT $2.2 \mu \mathrm{m}$ & $\begin{array}{c}R b m 20^{\Delta R R M} \\
1.9 \mu \mathrm{m}\end{array}$ & $\begin{array}{c}R b m 20^{\Delta R R M} \\
2.2 \mu \mathrm{m}\end{array}$ \\
\hline$k_{-A D P}\left(s^{-1}\right)$ & $189.64 \pm 12.42$ & $167.83 \pm 7.16$ & $164.41 \pm 12.27^{\ddagger}$ & $128.75 \pm 7.33^{\dagger *}$ \\
\hline $\mathrm{k}_{+\mathrm{ATP}}\left(\mathrm{mM}^{-1} \mathrm{~s}^{-1}\right)$ & $1225.46 \pm 193.46$ & $842.04 \pm 123.64$ & $1132.56 \pm 134.14$ & $988.29 \pm 177.37$ \\
\hline
\end{tabular}

$k_{-A D P}$, cross-bridge MgADP release rate.

$k_{+}$ATP, cross-bridge MgATP binding rate.

${ }^{+} p<0.05,{ }^{\ddagger} p<0.1$ effect of mutation at same sarcomere lengths.

${ }^{*} p<0.05$ effect of sarcomere length under similar treatment conditions.

$\mu \mathrm{m}$ sarcomere length in $R b m 20^{\triangle R R M}$ strips, compared to WT strips, which would be expected to slow cross-bridge detachment and stabilize, or amplify thin-filament activation more greatly in $R b m 20^{\triangle R R M}$ strips. However, slower cross-bridge detachment rates did not enhance tension nor length-dependent activation of contraction in $R b m 20^{\triangle R R M}$ strips with greater titin compliance. Thus, cross-bridge contributions to thin-filament activation and increased $\mathrm{Ca}^{2+}$-affinity of troponin $\mathrm{C}$ may require titin interacting with the thin-filament or titin transmitting tension between the thick and thin-filament. Increased titin compliance in $R b m 20^{\triangle R R M}$ strips may compromise this titin interaction or tension transmission pathway, thereby depressing $\mathrm{Ca}^{2+}$. activated tension production and length-dependent activation of contraction.

Some muscle mechanics studies use large amplitude releaserestretch protocols $(\sim 15 \%$ muscle length) to assess the crossbridge rate of tension redevelopment $\left(k_{t r}\right)$, in comparison to the low amplitude strains used for stochastic length perturbation analysis ( $<0.15 \%$ muscle length). Skinned myocardial strips from $\mathrm{WT}$ and $R b m 20^{\triangle R R M}$ strips mice showed no differences in sarcomere length-dependent $k_{t r}$ under maximally $\mathrm{Ca}^{2+}$-activated conditions (Methawasin et al., 2014). Previous studies using skinned myocardium from rats expressing the more compliant N2BA titin isoform have shown mixed reports of slower and faster $k_{t r}$ values as sarcomere length increased (Patel et al., 2012; Hanft et al., 2014), compared to wild-type controls that predominantly express the stiffer N2B titin isoform. Herein we measured cross-bridge kinetics as [MgATP] varied, which allowed us to estimate cross-bridge rates of MgADP release $\left(k_{-A D P}\right)$ or MgATP binding $\left(k_{+A T P}\right.$; Table 2$)$. As the rate of MgADP release limits cross-bridge detachment in a muscle fiber (Siemankowski et al., 1985), the $\sim 12 \%$ slowing in $k_{-A D P}$ from 1.9 to $2.2 \mu \mathrm{m}$ sarcomere length drives the length-dependent slowing of cross-bridge detachment in WT strips. However, the length-dependent slowing of $k_{-A D P}$ was nearly twice as great in $R b m 20^{\triangle R R M}$ strips $(\sim 22 \%)$ and $k_{-A D P}$ was also slower at each sarcomere length when titin compliance increased. There was not a significant difference between cross-bridge MgATP binding rates at 1.9 vs. $2.2 \mu \mathrm{m}$ sarcomere lengths for either genotype. These data support our previous observations that slowed MgADP release rate is the predominate step of the cross-bridge cycle that is responsible for the length-dependent slowing of cross-bridge kinetics (Tanner et al., 2015; Pulcastro et al., 2016). We do not think these slowed nucleotide handling kinetics in $R b m 20^{\triangle R R M}$ strips stem from any $\alpha$-to- $\beta$ myosin heavy chain isoform shift, because Methawasin et al. (2014) reported solely $\alpha$-myosin heavy chain expression in both of these mouse lines. Moreover, these data also demonstrate that titin compliance influences sarcomere length-dependent cross-bridge nucleotide handling rates, and the effects of titin on cross-bridge kinetics become greater as sarcomere length increases.

Under relaxed conditions, both viscoelastic mechanical stiffness (Figure 2) and steady-state tension values (Table 1) were greater at longer sarcomere length, without any differences between the two genotypes. These differences stem from passive elements of the sarcomere being stretched or extended more greatly at 2.2 vs. $1.9 \mu \mathrm{m}$ sarcomere length [i.e., titin and collagen (Granzier and Irving, 1995)]. We had anticipated that lengthdependent increases in relaxed stiffness and tension would be greater for WT vs. Rbm $20^{\triangle R R M}$, similar to previous observations using skinned myocytes (Methawasin et al., 2014). However, Methawasin et al. (2014) also showed greater collagen expression in $R b m 20^{\triangle R R M}$ vs. heterozygous $R b m 20$ knockout mice, which could be a compensatory mechanism to increase myocardial stiffness as titin compliance decreased in the homozygous mice. Given that skinned myocardial strips encompass some component of passive stiffness due to collagen that isn't present in isolated myocytes, it is possible that the mechanical characteristics of collagen, rather than titin, are dominating our relaxed muscle mechanics measurements. While previous studies suggested that greater passive tension values are correlated with greater $\mathrm{Ca}^{2+}$-activated tension production and length-dependent activation of contraction (Fukuda et al., 2001, 2003), our 
measurements do not support this mechanism driving lengthdependent activation because relaxed stiffness and tension values were similar at each sarcomere length for both genotypes.

Thick-to-thin-filament spacing consistently decreases as sarcomere length increases in skinned and intact muscle preparations (Matsubara and Millman, 1974; Irving et al., 2000; Konhilas et al., 2002; Smith et al., 2009). Mechanical characteristics of titin influence this lattice spacing vs. sarcomere length relationship, showing that increased titin compliance can increase myofilament lattice spacing and affect the relationship between lattice spacing and sarcomere length (both increasing and decreasing the slope of this relationship; Cazorla et al., 2001; Fukuda et al., 2001, 2003, 2005; Irving et al., 2011). In addition, recent measurements show smaller myofilament lattice spacing values in $R b m 20$ knockout rat myocardium at both short and long sarcomere length, compared to wild-type controls (Ait-Mou et al., 2016). Cross-bridge cycling rates have been shown to slow as thick-to-thin-filament spacing decreased in vertebrate and invertebrate muscle fibers that were osmotically compressed with Dextran (Krasner and Maughan, 1984; Kawai and Schulman, 1985; Smith et al., 2009; Tanner et al., 2012b) and with increases in sarcomere length in skinned (Adhikari and Wang, 2004; Tanner et al., 2015; Pulcastro et al., 2016), and intact (Milani-Nejad et al., 2013) cardiac muscle preparations. Therefore, increases in sarcomere length will accompany decreases in thick-to-thinfilament spacing, which could contribute to slower cross-bridge detachment at longer sarcomere length for both genotypes.

While reduced lattice spacing may slow cross-bridge cycling, this does not translate into increased length-dependent activation of contraction in $R b m 20^{\triangle R R M}$ strips (Hanft et al., 2014; Methawasin et al., 2014). Therefore, slowed cross-bridge cycling kinetics may not be the primary mechanism responsible for increasing $\mathrm{Ca}^{2+}$-activated tension at long sarcomere length (Patel et al., 2012), particularly when titin compliance increases from normal. Perhaps, titin interacts with the thin-filament to influence thin-filament activation and length-dependent

\section{REFERENCES}

Adhikari, B., and Wang, K. (2004). Interplay of troponin- and myosin-based pathways of calcium activation in skeletal and cardiac muscle: the use of W7 as an inhibitor of thin filament activation. Biophys. J. 86, 359-370. doi: 10.1016/S0006-3495(04)74112-0

Ait-Mou, Y., Hsu, K., Farman, G. P., Kumar, M., Greaser, M. L., Irving, T. C., et al. (2016). Titin strain contributes to the Frank-Starling law of the heart by structural rearrangements of both thin- and thick-filament proteins. Proc. Natl. Acad. Sci. U.S.A. 113, 2306-2311. doi: 10.1073/pnas.15167 32113

Bremel, R. D., and Weber, A. (1972). Cooperation within actin filament in vertebrate skeletal muscle. Nat. New Biol. 238, 97-101. doi: 10.1038/newbio238097a0

Campbell, K. (2006). Filament compliance effects can explain tension overshoots during force development. Biophys. J. 91, 4102-4109. doi: 10.1529/biophysj.106.087312

Campbell, K., Chandra, M., Kirkpatrick, R., Slinker, B., and Hunter, W. (2004). Interpreting cardiac muscle force-length dynamics using a novel functional model. Am. J. Physiol. Heart Circ. Physiol. 286, H1535-H1545. doi: 10.1152/ajpheart.01029.2003 activation of contraction, either directly or by influencing load (or strain) borne by thin-filament proteins (Terui et al., 2008; Hanft et al., 2014). This titin-thin-filament activation pathway may be suppressed with the more compliant titin in $R b m 20^{\triangle R R M}$ fibers, because titin is less taut and cannot effectively transmit tension between the M-band and Z-disks to maintain tension throughout the sarcomere. Thus, increases in cross-bridge duty ratio due to slowed detachment kinetics in $R b m 20^{\triangle R R M}$ fibers do not necessarily translate into the greater tension production due to a compromised capacity to generate tension or distribute tension throughout a more compliant sarcomere. Altogether, this would diminish ventricular function, and may scale with the expression ratio between the more compliant N2BA titin isoform and the stiffer N2B titin isoform. These impaired mechanisms of thin-filament activation and tension production may contribute to cardiac dysfunction and the associated cardiomyopathies in humans, rats, and mice bearing RBM20 mutations that influence titin splicing (Makarenko et al., 2004; Nagueh et al., 2004; Guo et al., 2012; Methawasin et al., 2014).

\section{AUTHOR CONTRIBUTIONS}

$\mathrm{HP}, \mathrm{PA}, \mathrm{MM}$, and BT participated in performing the experiments and data collection. BT, WD, and HG conceived and designed the experiments. HP, PA, and BT analyzed the data. All authors helped interpret the data, write, and revise the manuscript, and have approved the final version of this manuscript.

\section{ACKNOWLEDGMENTS}

This research was supported by Beginning Grant in Aide 14BGIA20380385 from the Western States Affiliate of the American Heart Association (BT), a New-faculty Seed Grant from the College of Veterinary Medicine (BT), and National Institutes of Health Grants R01HL118524 (HG), R01HL80186 (WD), and R21HL109693 (WD).
Campbell, K. S. (2009). Interactions between connected half-sarcomeres produce emergent mechanical behavior in a mathematical model of muscle. PLoS Comput. Biol. 5:e1000560. doi: 10.1371/journal.pcbi.1000560

Campbell, K. S. (2016). Compliance accelerates relaxation in muscle by allowing myosin heads to move relative to actin. Biophys. J. 110, 661-668. doi: 10.1016/j.bpj.2015.12.024

Cazorla, O., Wu, Y., Irving, T. C., and Granzier, H. (2001). Titin-based modulation of calcium sensitivity of active tension in mouse skinned cardiac myocytes. Circ. Res. 88, 1028-1035. doi: 10.1161/hh1001.090876

Chase, P. B., Macpherson, J. M., and Daniel, T. L. (2004). A spatially explicit nano-mechanical model of the half-sarcomere: myofilament compliance affects Ca2+ activation. Ann. Biomed. Eng. 32, 1556-1565. doi: 10.1114/B:ABME.0000049039.89173.08

Cooke, R. (1997). Actomyosin interaction in striated muscle. Physiol. Rev. 77, 671-697.

Daniel, T. L., Trimble, A. C., and Chase, P. B. (1998). Compliant realignment of binding sites in muscle: transient behavior and mechanical tuning. Biophys. J. 74, 1611-1621. doi: 10.1016/S0006-3495(98)77875-0

Fitzsimons, D. P., and Moss, R. L. (1998). Strong binding of myosin modulates length-dependent $\mathrm{Ca} 2+$ activation of rat ventricular myocytes. Circ. Res. 83, 602-607. doi: 10.1161/01.RES.83.6.602 
Freiburg, A., and Gautel, M. (1996). A molecular map of the interactions between titin and myosin-binding protein C. Implications for sarcomeric assembly in familial hypertrophic cardiomyopathy. Eur. J. Biochem. 235, 317-323. doi: 10.1111/j.1432-1033.1996.00317.x

Fukuda, N., Sasaki, D., Ishiwata, S., and Kurihara, S. (2001). Length dependence of tension generation in rat skinned cardiac muscle: role of titin in the Frank-Starling mechanism of the heart. Circulation 104, 1639-1645. doi: 10.1161/hc3901.095898

Fukuda, N., Wu, Y., Farman, G., Irving, T., and Granzier, H. (2003). Titin isoform variance and length dependence of activation in skinned bovine cardiac muscle. J. Physiol. 553, 147-154. doi: 10.1113/jphysiol.2003.049759

Fukuda, N., Wu, Y., Farman, G., Irving, T., and Granzier, H. (2005). Titinbased modulation of active tension and interfilament lattice spacing in skinned rat cardiac muscle. Pflugers Arch. Eur. J. Physiol. 449, 449-457. doi: 10.1007/s00424-004-1354-6

Godt, R. E., and Lindley, B. D. (1982). Influence of temperature upon contractile activation and isometric force production in mechanically skinned muscle fibers of the frog. J. Gen. Physiol. 80, 279-297. doi: 10.1085/jgp.80.2.279

Gordon, A. M., Homsher, E., and Regnier, M. (2000). Regulation of contraction in striated muscle. Physiol. Rev. 80, 853-924. Available online at: http://physrev. physiology.org/content/80/2/853.long

Granzier, H. L., and Irving, T. C. (1995). Passive tension in cardiac muscle: contribution of collagen, titin, microtubules, and intermediate filaments. Biophys. J. 68, 1027-1044. doi: 10.1016/S0006-3495(95)80278-X

Guo, W., Pleitner, J., and Greaser, M. (2013). Pathophysiological defects and transcriptional profiling inmodel RBM20-/- rat model. PLOS ONE 8:e84281. doi: 10.1371/journal.pone.0084281

Guo, W., Schafer, S., Greaser, M., Radke, M., Liss, M., Govindarajan, T., et al. (2012). RBM20, a gene for hereditary cardiomyopathy, regulates titin splicing. Nat. Med. 18, 766-773. doi: 10.1038/nm.2693

Hanft, L. M., Greaser, M. L., and McDonald, K. S. (2014). Titin-mediated control of cardiac myofibrillar function. Arch. Biochem. Biophys. 552-553, 83-91. doi: 10.1016/j.abb.2013.11.005

Helmes, M., Trombitas, K., and Granzier, H. (1996). Titin develops restoring force in rat cardiac myocytes. Circ. Res. 79, 619-626. doi: 10.1161/01.RES.79.3.619

Hofmann, P. A., and Fuchs, F. (1987). Effect of length and cross-bridge attachment on Ca2+ binding to cardiac troponin C. Am. J. Physiol. 253, C90-C96.

Irving, T. C., Konhilas, J., Perry, D., Fischetti, R., and de Tombe, P. P. (2000). Myofilament lattice spacing as a function of sarcomere length in isolated rat myocardium. Am. J. Physiol. Heart Circ. Physiol. 279, H2568-H2573. Available online at: http://ajpheart.physiology.org/content/279/5/H2568.long

Irving, T., Wu, Y., Bekyarova, T., Farman, G. P., Fukuda, N., and Granzier, H. (2011). Thick-filament strain and interfilament spacing in passive muscle: effect of titin-based passive tension. Biophys. J. 100, 1499-1508. doi: 10.1016/j.bpj.2011.01.059

Kawai, M., and Halvorson, H. (1991). Two step mechanism of phosphate release and the mechanisms of force generation in chemically skinned fibers of rabbit psoas muscle. Biophys. J. 59, 329-342. doi: 10.1016/S0006-3495(91)82227-5

Kawai, M., and Schulman, M. I. (1985). Crossbridge kinetics in chemically skinned rabbit psoas fibres when the actin-myosin lattice spacing is altered by dextran T-500. J. Muscle Res. Cell Motil. 6, 313-332. doi: 10.1007/BF00713172

Kobirumaki-Shimozawa, F., Inoue, T., Shintani, S. A., Oyama, K., Terui, T., Minamisawa, S., et al. (2014). Cardiac thin filament regulation and the Frank-Starling mechanism. J. Physiol. Sci. 64, 221-232. doi: 10.1007/s12576014-0314-y

Konhilas, J. P., Irving, T. C., and de Tombe, P. P. (2002). Myofilament calcium sensitivity in skinned rat cardiac trabeculae: role of interfilament spacing. Circ. Res. 90, 59-65. doi: 10.1161/hh0102.102269

Krasner, B., and Maughan, D. W. (1984). The relationship between ATP hydrolysis and active force in compressed and swollen skinned muscle fibers of the rabbit. Pflugers Arch. 400, 160-165. doi: 10.1007/BF00585033

Labeit, S., and Kolmerer, B. (1995). Titins: giant proteins in charge of muscle ultrastructure and elasticity. Science 270, 293-296. doi: 10.1126/science.270.5234.293

Lewinter, M. M., Popper, J., McNabb, M., Nyland, L., Bell, S. B., and Granzier, H. (2010). Extensible behavior of titin in the miniswine left ventricle. Circulation 121, 768-774. doi: 10.1161/CIRCULATIONAHA.109.918151
LeWinter, M., Wu, Y., Labeit, S., and Granzier, H. (2007). Cardiac titin: structure, functions, and role in disease. Clin. Chim. Acta 375, 1-9. doi: 10.1016/j.cca.2006.06.035

Li, K. L., Rieck, D., Solaro, R. J., and Dong, W. (2014). In situ time-resolved FRET reveals effects of sarcomere length on cardiac thin-filament activation. Biophys. J. 107, 682-693. doi: 10.1016/j.bpj.2014.05.044

Li, S., Guo, W., Dewey, C. N., and Greaser, M. L. (2013). Rbm20 regulates titin alternative splicing as a splicing repressor. Nucleic Acids Res. 41, 2659-2672. doi: $10.1093 /$ nar/gks1362

Lymn, R. W., and Taylor, E. W. (1971). Mechanism of adenosine triphosphate hydrolysis by actomyosin. Biochemistry 10, 4617-4624. doi: 10.1021/bi00801a004

Makarenko, I., Optiz, C., Leake, M., Neagoe, C., Kulke, M., Gwathmey, J., et al. (2004). Passive stiffness changes caused by upregulation of compliant titin isoforms in human dilated cardiomyopathy hearts. Circ. Res. 95, 708-716. doi: 10.1161/01.RES.0000143901.37063.2f

Martyn, D., Chase, P., Regnier, M., and Gordon, A. (2002). A simple model with myofilament compliance predicts activation-dependent crossbridge kinetics in skinned skeletal fibers. Biophys. J. 83, 3425-3434. doi: 10.1016/S00063495(02)75342-3

Matsubara, I., and Millman, B. M. (1974). X-ray diffraction patterns from mammalian heart muscle. J. Mol. Biol. 82, 527-536. doi: 10.1016/00222836(74)90246-0

Methawasin, M., Hutchinson, K., Lee, E., Smith, I. I. I. J., Saripalli, C., Hidalgo, C., et al. (2014). Experimentally increasing titin compliance in a novel mouse model attenuates the Frank-Starling mechanism but has a beneficial effect on diastole. Circ. Res. 129, 1924-1936. doi: 10.1161/CIRCULATIONAHA.113.005610

Metzger, J. M. (1995). Myosin binding-induced cooperative activation of the thin filament in cardiac myocytes and skeletal muscle fibers. Biophys. J. 68, 1430-1442. doi: 10.1016/S0006-3495(95)80316-4

Milani-Nejad, N., Xu, Y., Davis, J. P., Campbell, K. S., and Janssen, P. M. L. (2013). Effect of muscle length on cross-bridge kinetics in intact cardiac trabeculae at body temperature. J. Gen. Physiol. 141, 133-139. doi: 10.1085/jgp.2012 10894

Mulieri, L. A., Barnes, W. D., Leavett, B. J., Ittleman, F., LeWinter, M. M., Alpert, N. R., et al. (2002). Alterations of myocardial dynamic stiffness implicating abnormal crossbridge function in human mitral regurgitation heart failure. Circ. Res. 90, 66-72. doi: 10.1161/hh0102.103221

Nagueh, S., Shah, G., Wu, Y., Torre-Amione, G., King, N., Lahmers, S., et al. (2004). Altered titin expression, myocardial stiffness, and left ventricular function in patients with dilated cardiomyopathy. Circulation 110, 155-162. doi: 10.1161/01.CIR.0000135591.37759.AF

Palmer, B. M., Schmitt, J. P., Seidman, C. E., Seidman, J. G., Wang, Y., Bell, S. P., et al. (2013). Elevated rates of force development and MgATP binding in F764L and S532P myosin mutations causing dilated cardiomyopathy. J. Mol. Cell. Cardiol. 57, 23-31. doi: 10.1016/j.yjmcc.2012.12.022

Palmer, B. M., Suzuki, T., Wang, Y., Barnes, W. D., Miller, M. S., and Maughan, D. W. (2007). Two-state model of acto-myosin attachment-detachment predicts C-process of sinusoidal analysis. Biophys. J. 93, 760-769. doi: 10.1529/biophysj.106.101626

Patel, J. R., Pleitner, J. M., Moss, R. L., and Greaser, M. L. (2012). Magnitude of length-dependent changes in contractile properties varies with titin isoform in rat ventricles. Am. J. Physiol. Heart Circ. Physiol. 302, H697-H708. doi: 10.1152/ajpheart.00800.2011

Pulcastro, H. C., Awinda, P. O., Breithaupt, J. J., and Tanner, B. C. W. (2016). Effects of myosin light chain phosphorylation on length-dependent myosin kinetics in skinned rat myocardium. Arch. Biochem. Biophys. 601, 56-68. doi: 10.1016/j.abb.2015.12.014

Sheikh, F., Ouyang, K., Campbell, S. G., Lyon, R. C., Chuang, J., Fitzsimons, D., et al. (2012). Mouse and computational models link Mlc2v dephosphorylation to altered myosin kinetics in early cardiac disease. J. Clin. Invest. 122, 1209-1221. doi: 10.1172/JCI61134

Siemankowski, R. F., Wiseman, M. O., and White, H. D. (1985). ADP dissociation from actomyosin subfragment 1 is sufficiently slow to limit the unloaded shortening velocity in vertebrate muscle. Proc. Natl. Acad. Sci. U.S.A. 82, 658-662. doi: 10.1073/pnas.82.3.658 
Smith, L., Tainter, C., Regnier, M., and Martyn, D. A. (2009). Cooperative crossbridge activation of thin filaments contributes to the Frank-Starling mechanism in cardiac muscle. Biophys. J. 96, 3692-3702. doi: 10.1016/j.bpj.2009. 02.018

Tanner, B. C. W., Breithaupt, J. J., and Awinda, P. O. (2015). Myosin MgADP release rate decreases at longer sarcomere length to prolong myosin attachment time in skinned rat myocardium. Am. J. Physiol. Heart Circ. Physiol. 309, H2087-H2097. doi: 10.1152/ajpheart.0055 5.2015

Tanner, B. C. W., Daniel, T. L., and Regnier, M. (2012a). Filament compliance influences cooperative activation of thin filaments and the dynamics of force production in skeletal muscle. PLoS Comput. Biol. 8:e1002506. doi: 10.1371/journal.pcbi.1002506

Tanner, B. C. W., Farman, G. P., Irving, T. C., Maughan, D. W., Palmer, B. M., and Miller, M. S. (2012b). Thick-to-thin filament surface distance modulates cross-bridge kinetics in Drosophila flight muscle. Biophys. J. 103, 1275-1284. doi: 10.1016/j.bpj.2012.08.014

Tanner, B. C. W., McNabb, M., Palmer, B. M., Toth, M. J., and Miller, M. S. (2014). Random myosin loss along thick-filaments increases myosin attachment time and the proportion of bound myosin heads to mitigate force decline in skeletal muscle. Arch. Biochem. Biophys. 552-553, 117-127. doi: 10.1016/j.abb.2014.01.015

Tanner, B. C. W., Wang, Y., Maughan, D. W., and Palmer, B. M. (2011). Measuring myosin cross-bridge attachment time in activated muscle fibers using stochastic vs. sinusoidal length perturbation analysis. J. Appl. Physiol. 110, 1101-1108. doi: 10.1152/japplphysiol.00800.2010

Terui, T., Shimamoto, Y., Yamane, M., Kobirumaki, F., Ohtsuki, I., Ishiwata, S., et al. (2010). Regulatory mechanism of length-dependent activation in skinned porcine ventricular muscle: role of thin filament cooperative activation in the Frank-Starling relation. J. Gen. Physiol. 136, 469-482. doi: 10.1085/jgp.201010502

Terui, T., Sodnomtseren, M., Matsuba, D., Udaka, J., Ishiwata, S., Ohtsuki, I., et al. (2008). Troponin and titin coordinately regulate length-dependent activation in skinned porcine ventricular muscle. J. Gen. Physiol. 131, 275-283. doi: 10.1085/jgp.200709895

Tobacman, L. S. (1996). Thin filament-mediated regulation of cardiac contraction. Annu. Rev. Physiol. 58, 447-481. doi: 10.1146/annurev.ph.58.030196.002311
Tyska, M. J., and Warshaw, D. M. (2002). The myosin power stroke. Cell Motil. Cytoskeleton 51, 1-15. doi: 10.1002/cm.10014

Wang, L., Ji, X., Barefield, D., Sadayappan, S., and Kawai, M. (2014). Phosphorylation of cMyBP-C affects contractile mechanisms in a site-specific manner. Biophys. J. 106, 1112-1122. doi: 10.1016/j.bpj.2014.01.029

Wang, Y. P., and Fuchs, F. (1994). Length, force, and Ca(2+)-troponin C affinity in cardiac and slow skeletal muscle. Am. J. Physiol. 266, C1077-C1082.

Wang, Y., Tanner, B. C. W., Lombardo, A. T., Tremble, S. M., Maughan, D. W., Vanburen, P., et al. (2013). Cardiac myosin isoforms exhibit differential rates of MgADP release and MgATP binding detected by myocardial viscoelasticity. J. Mol. Cell. Cardiol. 54, 1-8. doi: 10.1016/j.yjmcc.2012.10.010

Weiss, K., Bottomley, P. A., and Weiss, R. G. (2015). On the theoretical limits of detecting cyclic changes in cardiac high-energy phosphates and creatine kinase reaction kinetics using in vivo 31P MRS. NMR Biomed. 28, 694-705. doi: 10.1002/nbm.3302

Wu, F., Zhang, E. Y., Zhang, J., Bache, R. J., and Beard, D. A. (2008). Phosphate metabolite concentrations and ATP hydrolysis potential in normal and ischaemic hearts. J. Physiol. 586, 4193-4208. doi: 10.1113/jphysiol.2008.154732

Wu, Y., Cazorla, O., Labeit, D., Labeit, S., and Granzier, H. (2000). Changes in titin and collagen underlie diastolic stiffness diversity of cardiac muscle. J. Mol. Cell. Cardiol. 32, 2151-2161. doi: 10.1006/jmcc.2000.1281

Zhao, Y., and Kawai, M. (1993). The effect of the lattice spacing change on cross-bridge kinetics in chemically skinned rabbit psoas muscle fibers. II Elementary steps affected by the spacing change. Biophys. J. 64, 197-210. doi: 10.1016/S0006-3495(93)81357-2

Conflict of Interest Statement: The authors declare that the research was conducted in the absence of any commercial or financial relationships that could be construed as a potential conflict of interest.

Copyright (c) 2016 Pulcastro, Awinda, Methawasin, Granzier, Dong and Tanner. This is an open-access article distributed under the terms of the Creative Commons Attribution License (CC BY). The use, distribution or reproduction in other forums is permitted, provided the original author(s) or licensor are credited and that the original publication in this journal is cited, in accordance with accepted academic practice. No use, distribution or reproduction is permitted which does not comply with these terms. 